\title{
Phase-inversion investigations of rubber-modified epoxies by electron microscopy and $\mathrm{X}$-ray diffraction
}

\author{
KING - FU LIN, UNG - LONG CHUNG \\ Institute of Materials Science and Engineering, National Taiwan University, Taipei, Taiwan \\ 10764
}

The phase-inversion phenomena of diglycidyl ether of bisphenol A (DGEBA) incorporated with a carboxy-terminated butadiene-acrylonitrile (CTBN) containing $17 \mathrm{wt} \%$ bound acrylonitrile after curing with ethylene diamine (EDA) in a stoichiometric ratio were investigated by scanning and transmission electron microscopy (SEM and TEM), and wideangle $X$-ray scattering (WAXS). The results of the SEM studies revealed that the rubber-rich phases in a size range of 1-30 $\mu \mathrm{m}$, which originally dispersed in an epoxy-rich matrix, gradually transformed to a co-continuous phase as the CTBN content reached $30 \mathrm{phr}$. As the CTBN content was increased to $50 \mathrm{phr}$, that phase inversion, as the continuous epoxy-rich phase transformed to a dispersed phase, took place. However, because the transformed dispersed phase was very small, the phase inversion could only be observed by TEM in specimens stained by osmium tetraoxide $\left(\mathrm{OsO}_{4}\right)$. On the other hand, we also found that the phase inversion could be detected by WAXS, and this showed the absence of a characterization peak of the epoxy resin at the scattering vector, $Q=4.1 \mathrm{~nm}^{-1}$. To the best of our knowledge, this was the first time in the literature that WAXS has been employed to detect a phase inversion.

\section{Introduction}

The wide-angle X-ray scattering (WAXS) of cured epoxy resins has a diffraction peak in the scatteringvector $(Q=4 \pi \sin (\theta / \lambda))$ range of $3.4-4.6 \mathrm{~nm}^{-1}$; this has been recently studied and it is well recognized as a characterization peak associated with the localordering structure in epoxy networks [1-3]. According to Bragg's equation, the $d$-spacing of the corresponding local-ordering structure is in the $1.4-1.9 \mathrm{~nm}$ range. In the fracture tests of chrome acetylactonatefortified, amine-cured epoxy resins in our laboratory, the top layer in certain areas of the fracture surface with thicknesses the same order of magnitude as the $d$ spacing of the local-ordering structure was fractured into pieces [4], indicating that the WAXS-detected local-ordering structure is a true structure. Thus, we considered that it might be possible to employ the characterization peak as an indicator to detect the phase inversion of rubber-modified epoxy resins.

Epoxy resins are brittle in nature. Research workers usually blend them with liquid rubber to improve their toughness. To optimize the toughness and other mechanical properties of toughened epoxy resins, workers also vary the rubber content, the type of hardener, the concentration of the hardener, the curing temperature, the initial molecular weight of the rubber, the choice of the end group on the rubber, etc., in the formulations [5-8]. However, although it has been reported that the fracture toughness of rubber- modified epoxy resins increased with the initial addition of small amounts of rubber [9], the reverse result of the toughness decreasing with increasing rubber content was found after the rubber content reached the level at which the phase inversion takes place [10]. Thus, finding an easy method for detecting phase inversion is important.

In this study, a diglycidyl ether of bisphenol $A$ (DGEBA) was mixed with various amounts of carboxy-terminated butadiene-acrylonitrile (CTBN) containing $17 \mathrm{wt} \%$ bound acrylonitrile before curing with ethylene diamine (EDA) in a stoichiometric ratio. Then, the phase-inversion phenomena were investigated by WAXS, and the results were compared to those from scanning and transmission electron microscopy (SEM and TEM).

\section{Experimental procedure}

\subsection{Materials}

The DGEBA was obtained from Shell with an epoxy equivalent weight (EEW) of $187 \pm 4 \mathrm{~g} \mathrm{eq}^{-1}$, measured by hydrochloric-acid-methyl-ethyl-ketone methods [11]. The CTBN was obtained from B. F. Goodrich containing $17 \mathrm{wt} \%$ bound acrylonitrile with a number-averaged molecular weight of $3500 \mathrm{~g} \mathrm{~mol}^{-1}$. The EDA curing agent was of reagent-grade; it was obtained from Osaka Chemical Industry in Japan. 


\subsection{Methods}

DGEBA and CTBN were preheated to $90^{\circ} \mathrm{C}$ and then mixed in various concentrations, by mechanical stirring, at the same temperature until the mixtures became transparent and then cooled to room temperature. The mixtures were added with the EDA curing agent in stoichiometric quantities with the epoxy groups and then they were degassed in a vacuum chamber. All the specimens were cured at $30^{\circ} \mathrm{C}$ for $24 \mathrm{~h}$ with $10 \mathrm{~h}$ at $128^{\circ} \mathrm{C}$ for the post-cure.

To prepare the fracture surface of cured specimens for SEM studies, the samples were cast and cured in a compact-tension-specimen (CTS) mould and fractured according to the method in [4]. The fracture surfaces obtained were gold-spattered and investigated in a Jeol JWM-5200 model scanning electron microscope. The specimens for TEM were cast and cured in capsules and then stained with osmium tetraoxide $\left(\mathrm{OsO}_{4}\right)$ prior to sectioning by an ultrathin microtome. A Jeol JEM-100U model transmission electron microscope was used to investigate the microstructure; the epoxy-rich phase appears as white areas, whereas the stained rubber-rich phase appears as darkened areas. The specimens for the WAXS studies were cast and cured in a rectangular mould with an inside dimension of $2.5 \times 15 \times 30 \mathrm{~mm}^{3}$. WAXS of the cured samples was performed on a Philips PW1710 model X-ray diffractometer using $\mathrm{CuK}_{\alpha}$ radiation and a graphite monochromator. The diffraction patterns were recorded by scanning at a rate of $3{ }^{\circ} \mathrm{C} \mathrm{min}{ }^{-1}$ over an angular range of $3^{\circ}<2 \theta<50^{\circ}$.

\section{Results and discussion}

\subsection{Electron microscopy of rubber-modified epoxies}

Fig. 1 shows SEM micrographs of fracture surfaces of the rubber-modified DGEBA/EDA epoxy resins incorporating 20,25, 30 and $50 \mathrm{phr}$ of CTBN liquid rubber. For the specimens incorporating 20 phr or less of rubber, rubber-rich domains were formed in spherical shapes $1-30 \mu \mathrm{m}$ in diameter; they were dispersed in an epoxy-rich matrix, as shown in Fig. 1a. However, as the rubber content was raised to $25 \mathrm{phr}$, the rubber particles coalesced and became irregularly shaped as shown in Fig. 1b. Furthermore, as the rubber content was increased to $30 \mathrm{phr}$, co-continuous phases with epoxy-rich and rubber-rich phases intermingled appeared in the structure, as shown in Fig. 1c. The rougher phases were a rubber-rich phase, whereas the smoother phases were an epoxy-rich phase as can be seen from the gradually growing rubber-rich phases shown in Fig. 1a to c. As the rubber content was increased to $50 \mathrm{phr}$, the rougher phase became the sole phase in the fracture surface as shown in Fig. 1d.

In order to investigate the origin of the roughness in the rubber-rich domains, the specimens were ultrathinly microtomed and stained with $\mathrm{OsO}_{4}$. A TEM micrograph of a stained rubber-modified epoxy resin
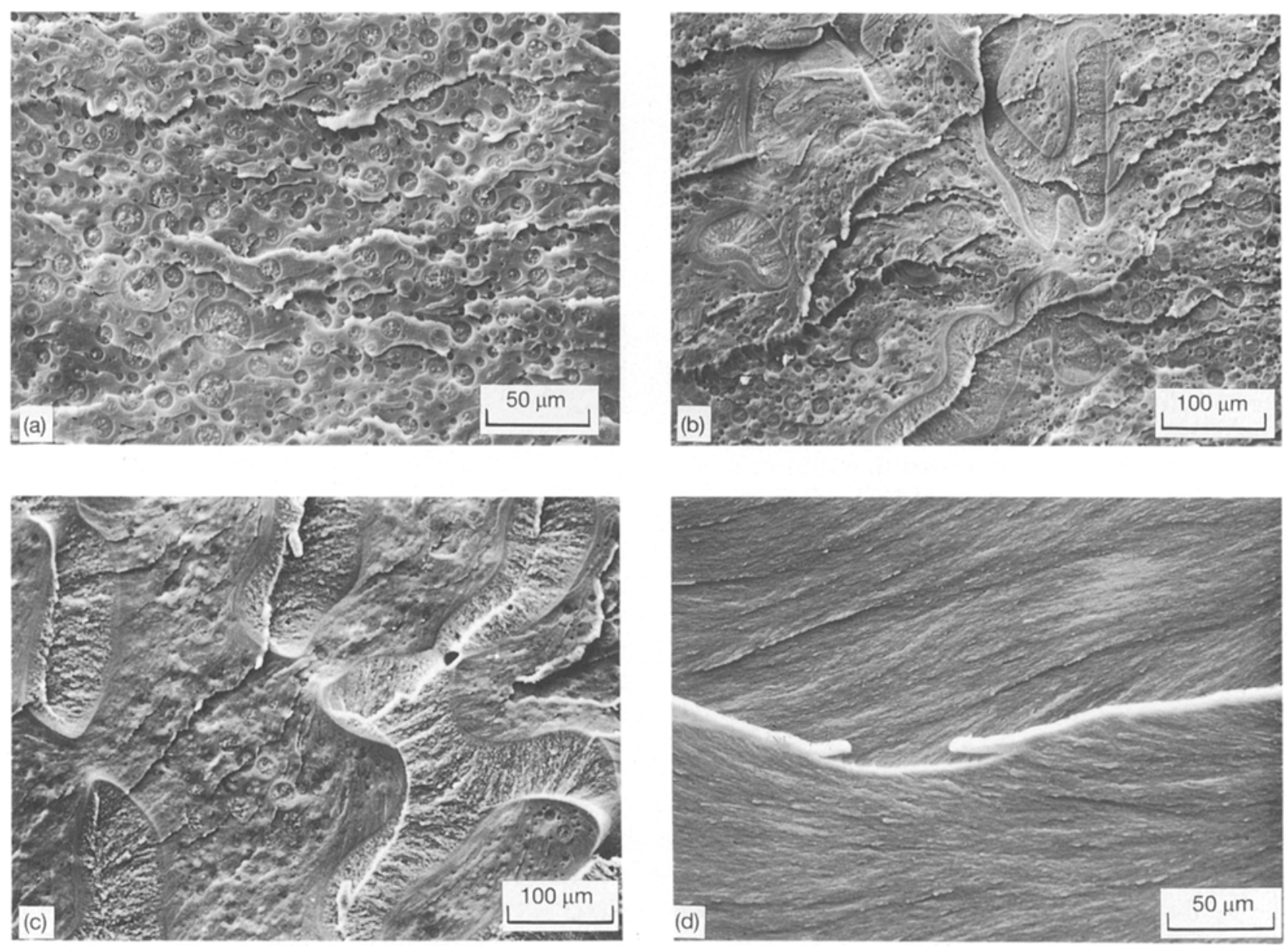

Figure I SEM micrographs of the fracture surfaces of rubber-modified DGEBA/EDA epoxy resins incorporating the following amounts of CTBN: (a) $20 \mathrm{phr}$, (b) $25 \mathrm{phr}$, (c) $30 \mathrm{phr}$, and (d) $50 \mathrm{phr}$. 

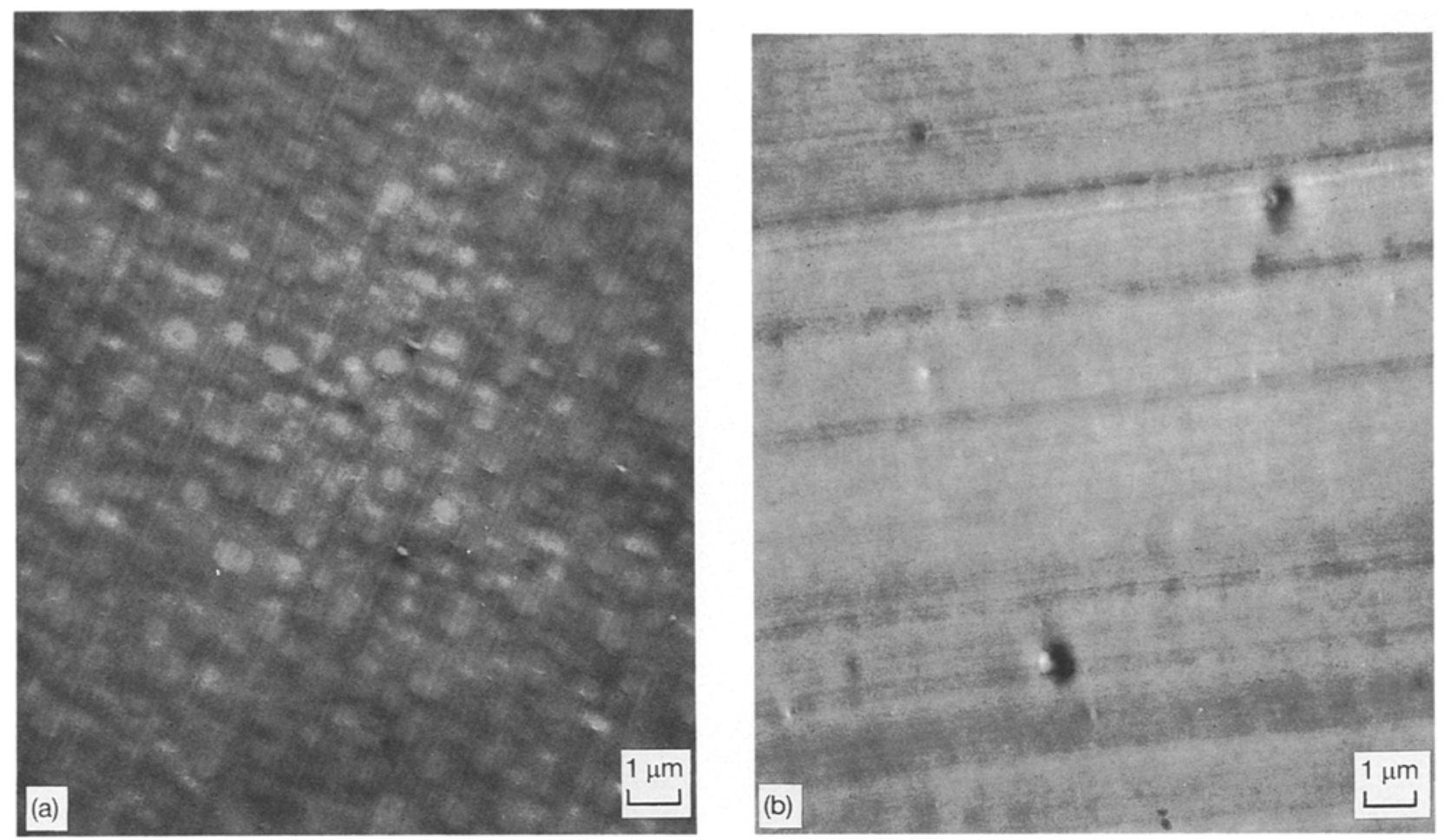

Figure 2 TEM micrographs of the $\mathrm{OsO}_{4}$-stained rubber-modified epoxy resins incorporating: (a) $50 \mathrm{phr}$, and (b) $60 \mathrm{phr}$ CTBN.

incorporating $50 \mathrm{phr}$ of CTBN is shown in Fig. $2 \mathrm{a}$, where the epoxy-rich phases appear as white particles with an average size of about $0.5 \mu \mathrm{m}$. These tiny epoxy-rich particles caused the roughness in the fracture surface observed by SEM. Obviously, the phaseinversion when the rubber-rich phases transformed to a continuous phase and when the epoxy-rich phases transformed to suspended phases occurred as the rubber-modified epoxy resin contains $50 \mathrm{phr}$ of CTBN. Moreover, tiny epoxy-rich particles were also found inside a rubber-rich domain in the epoxy resin incorporating $20 \mathrm{phr}$ CTBN, as shown in Figs 3 and 1a. As the rubber content was increased from 20 to $50 \mathrm{phr}$, the domains of the rubber-rich phase increased as the area of the continuous epoxy-rich phase decreased, whereas the tiny epoxy-rich particles barely changed their sizes and concentrations inside the rubber-rich domains but they did increase their total amount as the area of rubber-rich phase increased. As the rubber content was increased to 60 phr or more, the modified epoxy resins became homogeneous, and no significant microstructural features were observed by TEM, as shown in Fig. 2b. Obviously, the cured DGEBA/EDA epoxy resin is miscible with the CTBN after the rubber content reached $60 \mathrm{phr}$.

\subsection{X-ray scattering by rubber-modified epoxy resins}

The WAXS pattern of cured DGEBA/EDA epoxy resins has two diffraction peaks at the scattering vectors, $Q=4.1$ and $12.6 \mathrm{~nm}^{-1}$, as shown in Fig. 4. The peak at the small scattering vector is associated with the local-ordering structure in the epoxy networks, whereas the peak at the larger scattering vector is a typical amorphous halo arising from intermolecular correlations [1]. On the other hand, the WAXS of

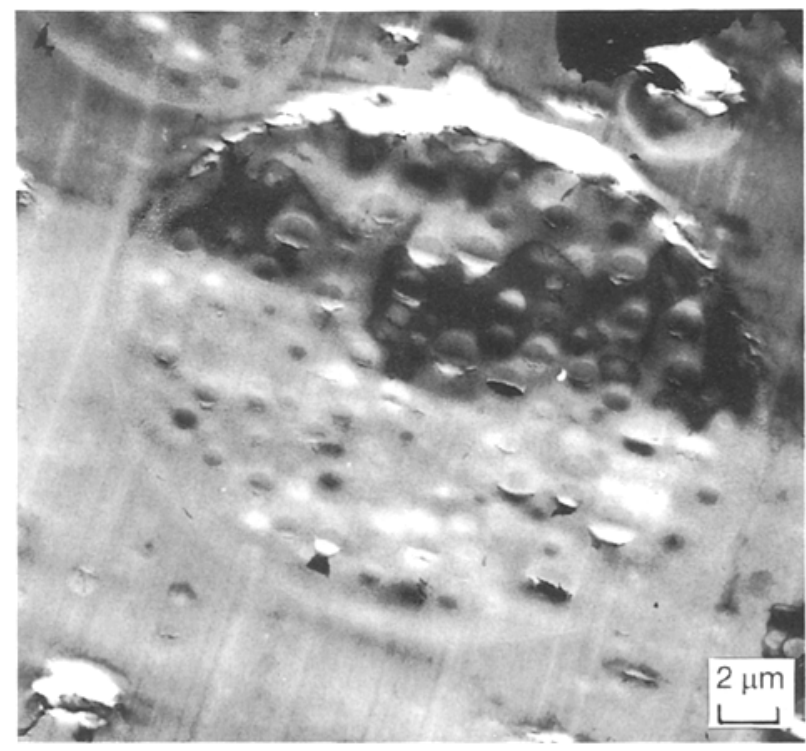

Figure 3 TEM photograph of $\mathrm{OsO}_{4}$-stained rubber-modified epoxy resin incorporating $20 \mathrm{phr}$ CTBN.

CTBN liquid rubber containing $17 \mathrm{wt} \%$ of bound acrylonitrile has an amorphous halo appeared at $13.7 \mathrm{~nm}^{-1}$ and a small-angle scattering, as shown in Fig. 4. A similar diffraction pattern was also reported for liquid argon by Eisenstein and Gingrich [12]; this is usually cited as an example of liquid studies.

Fig. 5 shows the WAXS patterns of modified DGEBA/EDA epoxy resins incorporating various amounts of the CTBN rubbers. Comparing with Fig. 4, the characterization peak at $Q=4.1 \mathrm{~nm}^{-1}$, associated with the local-ordering structure in the epoxy network, retained its position until the rubber content reached $50 \mathrm{phr}$, and it was completely smeared out at 50 phr. Roughly speaking, the WAXS patterns of rubber-modified epoxy resins were similar 


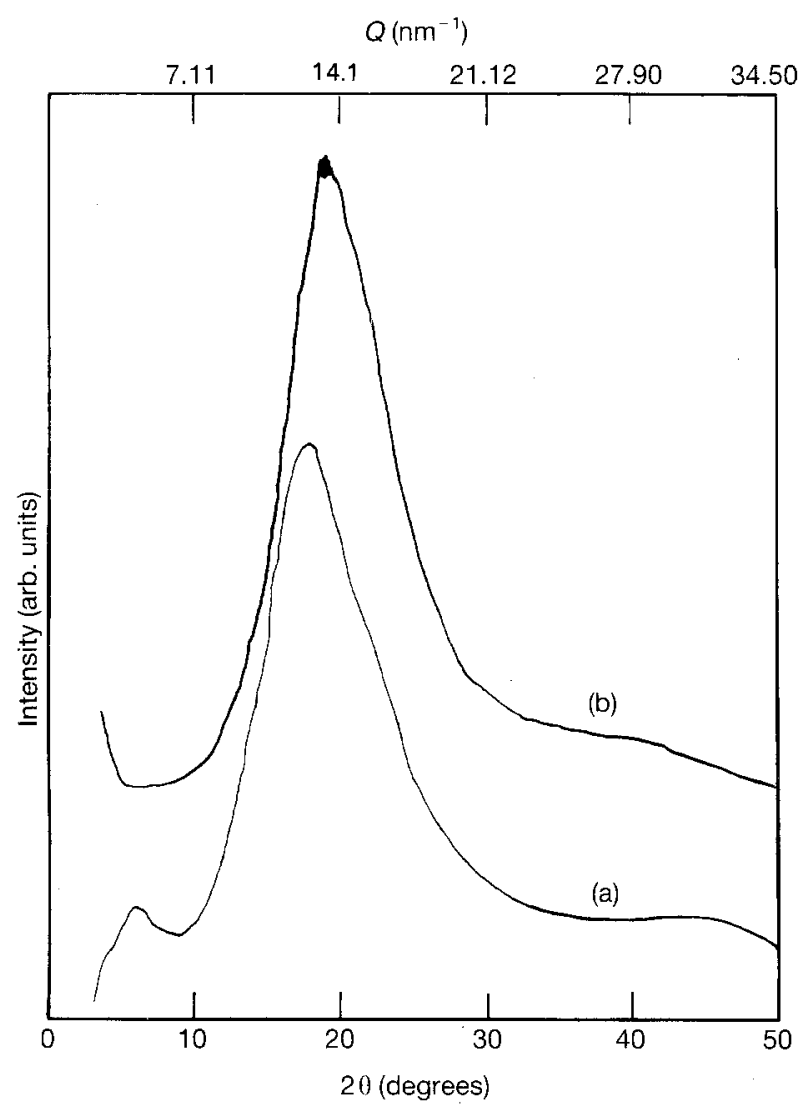

Figure 4 WAXS patterns of: (a) a cured DGEBA/EDA epoxy resin, and (b) a CTBN liquid rubber.

to those of the unmodified resins as long as the rubber content did not reach the level at which the phase inversion takes place. The disappearance of the characterization peak at the phase inversion indicates that the X-rays was mainly scattered by the continuous phase in the structure. After the rubber content reached $60 \mathrm{phr}$, the characterization peak appeared again in the same position, but its intensity was much lower than the intensity at rubber contents less than $50 \mathrm{phr}$ rubber, as can be seen in Fig. 5. Interestingly, the experimental results indicated that the content of epoxy resin in the matrix of the specimen containing $50 \mathrm{phr}$ of CTBN was less than that containing $60 \mathrm{phr}$.

Rubber-modified epoxy resins had a homogeneous structure after the rubber content reached $60 \mathrm{phr}$, whereas those incorporating $50 \mathrm{phr}$ rubber had epoxy-rich particles dispersed in a rubber-rich matrix, as shown in Fig. 2. According to the nucleationgrowth mechanism for the phase separation of a rubber-modified epoxy resin [13], the nucleation of the epoxy-rich domains has to overcome the surface tension between two phases, but the subsequent growth of the nucleated particles decreases the overall free energy. Thus, once the epoxy-rich particles are formed, as in the case of the modified epoxy resin incorporating $50 \mathrm{phr}$ of rubber, the amount of epoxy resin remaining in the matrix decreases until the growth rate is arrested. As a consequence, the concentration of epoxy resin in the rubber-rich matrix for the specimens containing $50 \mathrm{phr}$ of rubber is lower than that containing $60 \mathrm{phr}$. Therefore, the disappearance of the characterization peak in the WAXS pattern of

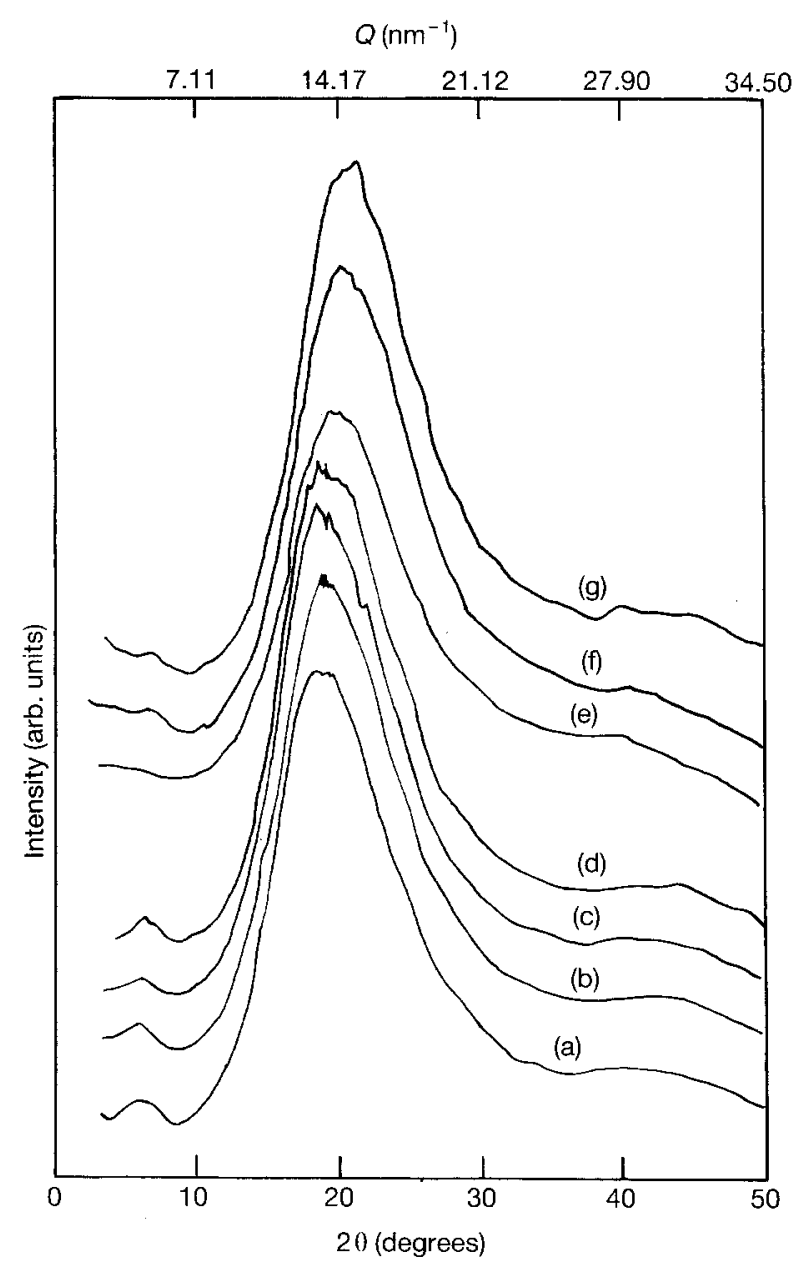

Figure 5 WAXS patterns of the rubber-modified epoxy resins incorporating the following amounts of CTBN: (a) $10 \mathrm{phr}$, (b) $20 \mathrm{phr}$, (c) $25 \mathrm{phr}$, (d) $30 \mathrm{phr}$, (e) $50 \mathrm{phr}$, (f) $60 \mathrm{phr}$ and (g) $70 \mathrm{phr}$.

the rubber-modified epoxy resin indicated the occurrence of the phase inversion, and a higher or lower resin content than that content giving rise to the phase inversion will generate the characterization peak. Although the most popular method of studying the phase inversion in rubber-modified epoxy resins is to directly observe the epoxy-rich domains by TEM, the WAXS technique is much easier and more reliable since direct observation by TEM requires a complicated sample preparation and it has limitations in the sample size and scope of view. After all, to our best knowledge, this is the first time in the literature that the WAXS technique has been employed in detecting the phase inversion of rubber-modified epoxy resins.

\section{Acknowledgement}

The authors wish to acknowledge financial support for this work from the National Science Council in Taiwan (grant number NSC80-0405-E002-12).

\section{References}

1. R. KUMAR and W. W. ADAMS, Polymer 28 (1987) 1497.

2. R. LOVELL and A. H. WINDLE, ibid. 31 (1990) 593.

3. V. P. KORKHOV, E. A. FAITEL'SON and Y. M. MOLCHANOV, Mek. Kompoz. Mater. 6 (1989) 1103.

4. K.-F. LIN, W.-Y. SHU and T.-L. WEY, Polymer, 34 (1993) 277. 
5. C. B. BUCKNALI. and T. YOSHI, Brit. Polym. J. 10 (1978) 53 .

6. J. N. SLitTAN and F. McGARRY, Polym. Engng. Sci. 13 (1973) 29.

7. C. K. RIEW, E. H. ROWE and A. R. SIEBERT, $A C S A d v$. Chem. Ser. 154 (1976) 326.

8. T. K. CHEN and Y. H. JAN, J. Mater. Sci. 27 (1992) 111

9. A. F. YEE and R. A. PEARSON, in "Fractography and failure mechanisms of polymers and composites", cdited by A. C. Roulin-Moloney (Elsevier Applicd Science, London, 1989) p. 291.

10. K.-F. LIN and W.-L. CHUNG, in preparation.
11. H. LEE and K. NEVILLE, in "Handbook of epoxy resins" (McGraw-Hill, New York, 1967) pp. 4-16

12. A. EISENSTEIN and N. S. GINGRICH, Phys. Ret. 58 (1940) 307.

13. A. VAZQUEZ, A. J. ROJAS, H. E. ADABBO, J. BORRAJO and R. J. J. WILLIAMS, Polymer 28 (1987) 1161.

Received 28 July 1992

and accepted 16 August 1993 